\title{
Wavelength conversion and supercontinuum generation in silicon optical fibers
}

\author{
Anna C. Peacock, Senior Member, IEEE, Joseph Campling, Antoine F. J. Runge, Haonan Ren, Li Shen, \\ Ozan Aktas, Peter Horak, Noel Healy, Ursula J. Gibson, and John Ballato, Fellow, IEEE
}

(Invited Paper)

\begin{abstract}
This paper describes the state-of-the-art in wavelength conversion and supercontinuum generation using glass-clad silicon core optical fibers. Such semiconductor fibers have enjoyed considerable attention due to their intrinsically high third-order nonlinearities, which are markedly higher than in conventional infrared glasses. Results to date from small core silicon fibers fabricated using both the high pressure chemical vapor deposition (HPCVD) technique and the molten core drawing (MCD) method are presented. Also discussed are directions for continued study and development, including engineering the dispersion and nonlinear properties as well as improved interconnection.
\end{abstract}

\section{INTRODUCTION}

S INCE their first realization a decade ago, glass-clad, Semiconductor core optical fibers have received attention as potential alternatives to soft glasses for infrared and nonlinear fiber optics [1]. Of the semiconductors fabricated into fibers to date, silicon is by far the most mature, with the fiber performance now rivaling that in planar analogs owing to considerable advancements in the materials optimization [2], [3], [4]. While transmission losses still need to be further reduced, preferably by an order of magnitude, and smaller core sizes achieved, the high third-order susceptibility of the silicon core material mark these fibers as particularly interesting for infrared sources based on wavelength conversion and supercontinuum generation.

This paper reviews progress in the studies of nonlinear propagation within the silicon core fibers. It begins with a brief discussion of the two methods employed to fabricate silicon optical fibers, including their relative advantages and disadvantages. From there, the nonlinear model used to describe light propagation in silicon fibers is presented, followed by experiments and numerical simulations to investigate wavelength conversion in fibers with amorphous and (poly)crystalline silicon core materials. Lastly, opportunities

A. C. Peacock, J. Campling, A. F. J. Runge, H. Ren, L. Shen, O. Aktas, and P. Horak are with the Optoelectronics Research Centre, University of Southampton, Southampton, SO17 1BJ, UK (e-mail:acp@orc.soton.ac.uk).

N. Healy is with the Physics Department, Emerging Technology and Materials Group, Newcastle University, Merz Court, Newcastle NE1 7RU, UK.

U. J. Gibson is with the Department of Physics, Norwegian University of Science and Technology, 7491 Trondheim, Norway.

J. Ballato is with the Center for Optical Materials Science and Engineering Technologies (COMSET) and Department of Materials Science and Engineering, Clemson University, Clemson, SC 29634, USA.

Manuscript received XXX, 2017. for future study to improve the efficiency of these fibers for nonlinear systems are provided.

\section{SiLICON FibER FABRICATION}

Fabricating practical optical fibers with either crystalline or amorphous semiconductor cores poses a significant materials science challenge due to the substantial thermal and thermomechanical mismatch with conventional glasses employed in more common fibers. For example, many semiconductors have melting points that are not in the same temperature range as the glass transition of fused silica, and/or do not melt congruently [5]. However, over the past 10 years two practical approaches to fabricating semiconductor optical fibers have emerged, the high pressure chemical vapor deposition (HPCVD) technique [6] and the molten core drawing (MCD) process [7]. Both methods have their merits and drawbacks, for example the HPCVD technique can be used to produce fibers with both amorphous and crystalline silicon cores, but is limited to fiber lengths of a few meters. Alternatively, the MCD process has so far been restricted to crystalline cores, but can be used to produce kilometers of fiber, considerably reducing the cost per length. Although at present the losses in these fibers limit the practical propagation lengths to a few centimeters, we expect that the current growth and development in their fabrication will eventually see the losses reduced to the $\mathrm{dB} / \mathrm{m}$ level [1]. Below we review these two complementary techniques in more detail.

\subsection{High Pressure Chemical Vapor Deposition}

The first silicon core fiber was produced by depositing the semiconductor material into the central pore of a silica capillary using the HPCVD method [8]. In this process, the capillary tubes have the dual function of acting as a reaction chamber for the deposition and as the low refractive index cladding material required for waveguiding. Prior to deposition, the silane precursor is mixed with an inert carrier gas to promote molecular transport through the capillary. The reaction is thermally initiated at the required location and, as the precursors decompose, the material is conformally deposited onto the capillary walls until the core is filled. Fig. 1(a) shows a scanning electron microscope (SEM) image 

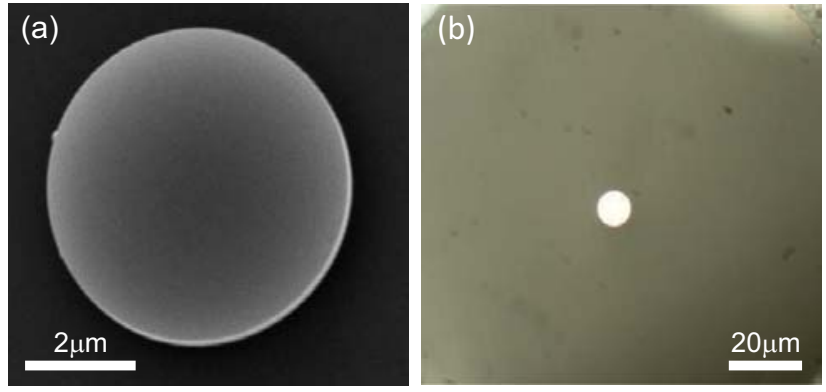

Fig. 1. Silicon core fibers fabricated via (a) the HPCVD approach and (b) the MCD method.

of a silicon fiber fabricated via this approach, confirming that the core consists of a solid silicon rod. Owing to the extremely smooth surfaces of the capillary templates (root mean square roughness $\sigma \sim 0.1 \mathrm{~nm}$ [9]) the core/cladding interface is near atomically smooth, which is particularly important for reducing scattering associated with the high index contrast, and thus for achieving low loss waveguides [10].

A key advantage of the HPCVD method is that the temperatures and pressures can be controlled to fill capillary dimensions ranging from several tens of micrometers down to much smaller, hundreds of nanometers, dimensions that are favoured for nonlinear applications. Furthermore, as the energy for precursor decomposition is significantly lower than that required for melting the bulk material, the process can be undertaken at relatively low temperatures, which reduces cracking and defect formation associated with the thermal mismatch of the materials [11]. However, significant disadvantages of this approach are that, owing to the small core sizes, the deposition rate is slow and it is difficult to control the grain growth when depositing in a crystalline form [12].

\subsection{Molten Core Drawing}

The MCD technique is based on a modified fiber drawing method, whereby the silica cladding acts as a crucible to contain the silicon as it is melted and drawn down to form the fiber core [13]. Owing to the practicality of the fiber drawing method, it is this approach that has been the most widely adopted by the fiber fabrication community [7], [14], [15], [16]. Typically, the fibers are drawn from a preform consisting of a silica tube filled with pure silicon, which can be in the form of a solid rod or powder. The preform is then heated to above both the glass transition temperature of the cladding and the melting temperature of the core. At this temperature the cladding glass can be deformed, but is still viscous enough to contain the molten silicon and maintain its cylindrical geometry when drawn. As the fiber leaves the furnace, it rapidly cools and the liquid core solidifies into a polycrystalline form, which is surrounded by the silica cladding. Fig. 1(b) displays a microscope image of a MCD derived silicon fiber, where the high index core is clearly visible with a well-defined core/cladding interface.

Key benefits of the MCD technique are that it can produce very long lengths of fiber and, by selecting the appropriate draw conditions, fibers with cores that consist of long (almost centimeter length) single crystal grains can be produced with relatively low optical losses [17], [18]. The main drawbacks of this method are associated with the high temperatures required to soften the cladding, which can cause oxygen to diffuse into the core and can induce significant cracking as the different materials cool. Although these issues can be somewhat mitigated via the introduction of a diffusion barrier around the core [15], as of to date, silicon core fibers fabricated using this method have been limited to core sizes that are several microns in diameter, or larger. Clearly the ability to obtain smaller core sizes is highly desirable for nonlinear applications and thus work in this area is ongoing.

\section{Nonlinear Propagation in Silicon Fibers}

As silicon is a uniform symmetric material, nonlinear transmission in these fibers is governed by processes involving the third order $\chi^{(3)}$ nonlinearity. The real part of the $\chi^{(3)}$ coefficient gives rise to the Kerr nonlinear refractive index $n_{2}$ and the imaginary part to the two photon absorption (TPA) parameter $\beta_{\text {TPA }}$. Thus pulse propagation in silicon core fibers can be described by a modified form of the nonlinear Schrödinger equation (NLSE) [19]:

$$
\begin{aligned}
& \frac{\partial A(z, t)}{\partial z}=-\frac{i \beta_{2}}{2} \frac{\partial^{2} A(z, t)}{\partial t^{2}}+\frac{\beta_{3}}{6} \frac{\partial^{3} A(z, t)}{\partial t^{3}} \\
& +i \gamma|A(z, t)|^{2} A(z, t)-\frac{1}{2}\left(\sigma_{f}+\alpha_{l}\right) A(z, t),
\end{aligned}
$$

where $A(z, t)$ is the pulse envelope, $\beta_{2}$ is the group velocity dispersion (GVD) parameter, $\beta_{3}$ is the third-order dispersion, $\gamma$ is the nonlinearity parameter, $\alpha_{l}$ is the linear loss, and $\sigma_{f}$ defines the free carrier contributions. Here, the nonlinear parameter is complex to account for both the Kerr and TPA contributions: $\gamma=k_{0} n_{2} / A_{\text {eff }}+i \beta_{\mathrm{TPA}} / 2 A_{\mathrm{eff}}$, where $A_{\mathrm{eff}}$ is the effective mode area. Similarly, the free carrier term is also complex $\sigma_{f}=\sigma(1+i \mu) N_{c}$, where $\sigma$ is the free carrier absorption (FCA) coefficient and $\mu$ governs the free carrier dispersion (FCD), and depends on the free carrier density $N_{c}$. The carrier density is related to the TPA parameter and can be determined via the rate equation [19]:

$$
\frac{\partial N_{c}(z, t)}{\partial t}=\frac{\beta_{\mathrm{TPA}}}{2 h \nu_{0}} \frac{|A(z, t)|^{4}}{A_{\mathrm{eff}}^{2}}-\frac{N_{c}(z, t)}{\tau_{c}},
$$

where $\tau_{c}$ is the carrier lifetime.

Compared to silica fibers, the silicon core material has a large nonlinearity and a high refractive index, which results in tight mode confinement, so that the nonlinear effects can be enhanced by several orders of magnitude [20]. As a result, propagation in the silicon fibers requires much lower pump powers (watts compared to kilowatts) and shorter fiber lengths (centimeters compared to kilometers) to observe efficient nonlinear wavelength conversion. However, the precise requirements depend on the nature of the silicon material and how it has been produced, which can alter both the linear and nonlinear parameters, as well as the fiber core size.

\section{HPCVD AMORPHOUS SILICON FIBERS}

The first observations of nonlinear propagation in silicon core fibers were recorded in a hydrogenated amorphous 
core material [21]. This is because, compared to crystalline silicon, hydrogenated amorphous silicon (a-Si:H) has a high Kerr nonlinear coefficient, on the order of $2-5$ times larger, as well as low linear and nonlinear optical losses [22]. The incorporation of hydrogen has played a crucial role in reducing the losses in this material as it passivates the dangling bonds, which are a major source of absorption in amorphous structures. The optical losses in this material have been significantly reduced over the last decade, from $\sim 20 \mathrm{~dB} / \mathrm{cm}$ down to $0.5 \mathrm{~dB} / \mathrm{cm}$, by controlling the concentration and distribution of hydrogen along the core [23]. Thus the precise values for the linear and nonlinear properties of this material depend on the hydrogen content, which can be tuned through the deposition parameters such as the precursor mixture and the temperature, as well as the operating wavelength. Typical values of the optical parameters for the HPCVD fibers fabricated from this material are shown in Table 1 for two pump wavelengths of interest; one in the telecoms band and one in the mid-infrared [23]. We note that these values are very similar to what has been reported for a-Si:H waveguides on-chip, indicating that the material quality is comparable [24].

TABLE 1

Material properties for a-Si:H core fibers.

\begin{tabular}{|c|c|c|}
\hline Pump $\lambda$ & $1.55 \mu \mathrm{m}$ & $2.4 \mu \mathrm{m}$ \\
\hline \hline$\alpha_{l}(\mathrm{~dB} / \mathrm{cm})$ & $0.9-3.5$ & $0.5-2$ \\
\hline$n_{2}\left(\times 10^{-13} \mathrm{~cm}^{2} / \mathrm{W}\right)$ & $1.5-1.85$ & $1.0-1.3$ \\
\hline$\beta_{\mathrm{TPA}}(\mathrm{cm} / \mathrm{GW})$ & $0.5-0.8$ & $0.01-0.03$ \\
\hline
\end{tabular}

As all of the initial nonlinear investigations of these fibers were conducted in structures with fairly large core diameters $(\sim 6 \mu \mathrm{m})$, where the dispersion properties are dominated by the large normal material dispersion (see Fig. 2), the observed spectral broadening was fairly modest [21]. Nevertheless, it was still possible to use these fibers for all-optical processes such as ultrafast modulation [25] and switching [26]. However, a significant breakthrough was made when low loss a-Si:H fibers with much smaller core diameters $(\sim 1-2 \mu \mathrm{m})$ were produced [3]. In this regard, reducing the core size had a two fold advantage; firstly, the nonlinearity was enhanced via the reduction in the mode area, and secondly, the increased waveguide

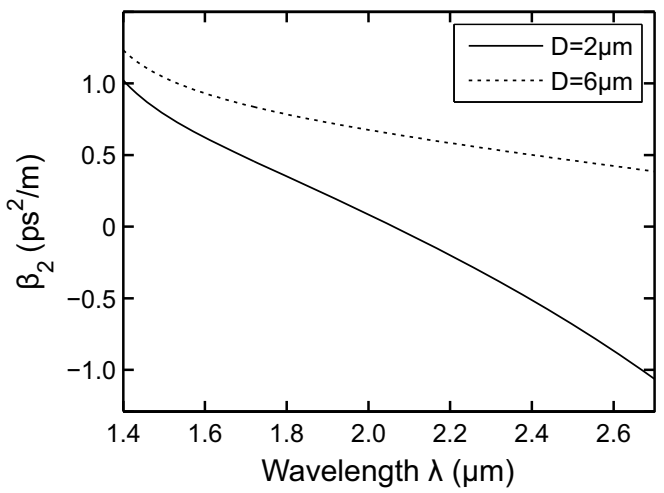

Fig. 2. Group velocity dispersion curves for two silicon fibers with micronsized core diameters (see legend).

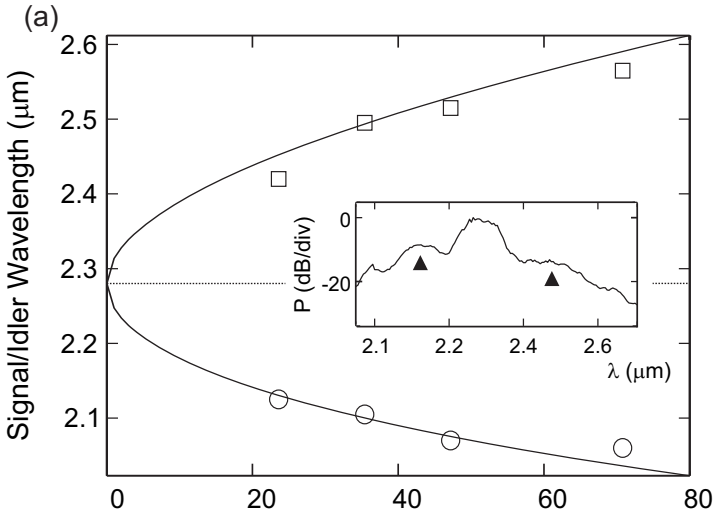

(b) Coupled peak power (W)

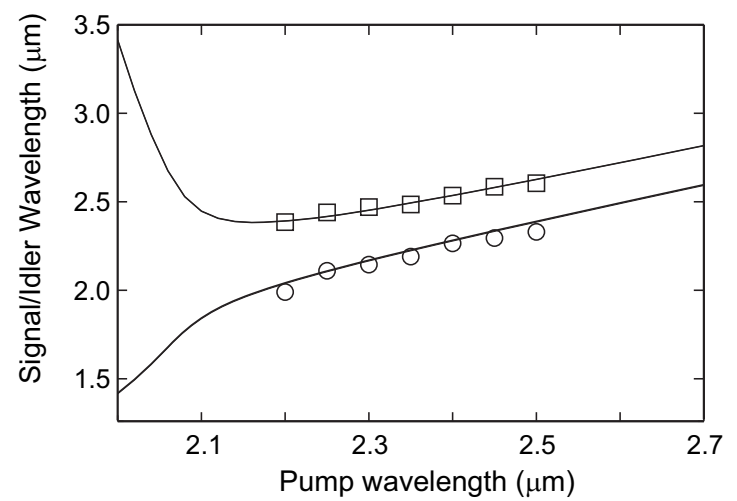

Fig. 3. (a) Peak FWM signal (circles) and idler (squares) wavelengths plotted as a function of coupled peak power for $\lambda_{p}=2.28 \mu \mathrm{m}$. Inset shows typical nonlinear broadening observed in an output spectrum, with the FWM sidebands indicated by up-arrows. (b) FWM signal (circles) and idler (squares) positions as a function of pump wavelength for $P_{0}=20 \mathrm{~W}$. In both cases the solid lines are the phase-matching predictions from $\Omega_{\max }$.

dispersion could compensate for the material dispersion to access the anomalous dispersion regime, as illustrated in Fig. 2. As a result, these small core fibers opened a route for the observation of nonlinear processes such as fourwave mixing (FWM) and soliton effects that are critical for generating broadband supercontinuum.

To demonstrate this, a $4 \mathrm{~mm}$ long a-Si:H fiber with a $1.7 \mu \mathrm{m}$ diameter core was pumped with a high power femtosecond (full width at half maximum $T_{\mathrm{FWHM}} \sim 300 \mathrm{fs}$ ) optical parametric oscillator (OPO) to investigate the process of spontaneous FWM. By tuning the OPO to operate on the edge of the anomalous dispersion regime $\left(\lambda_{p}=2.28 \mu \mathrm{m}\right)$, where optimum efficiency for the phase-matched process is expected, the characteristic FWM sidebands appeared on either side of the pump (see inset in Fig. 3(a)). In this region, the phase-matching condition can be well approximated by [27]:

$$
\beta_{2} \Omega^{2}+2 \operatorname{Re}[\gamma] P_{0}=0,
$$

where $P_{0}$ is the peak pump power and $\Omega=\left|\omega_{p}-\omega_{s}\right|=$ $\left|\omega_{p}-\omega_{i}\right|$ is the frequency shift between the pump and the signal/idler. Thus the position of the peak FWM gain can be estimated as:

$$
\Omega_{\max }= \pm \sqrt{2 \operatorname{Re}[\gamma] P_{0} /\left|\beta_{2}\right|} .
$$

Fig. 3 shows the measured tunability of the FWM peaks as a function of (a) pump power and (b) pump wavelength in 


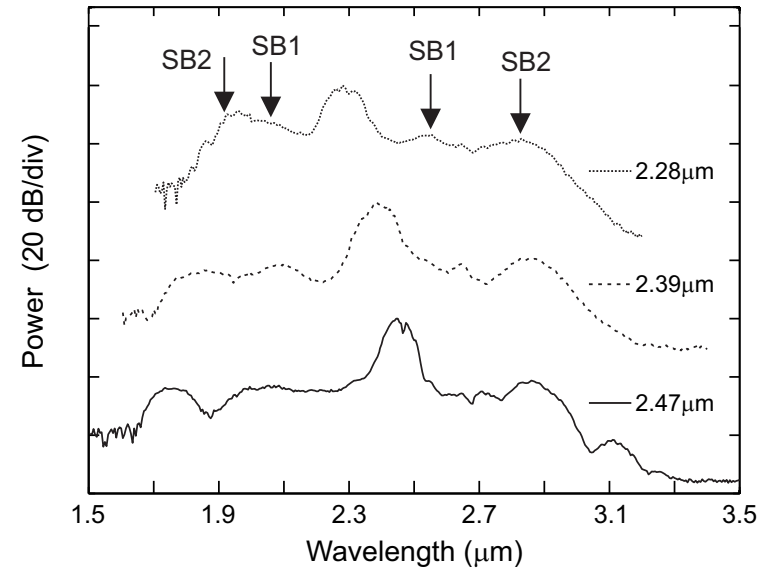

Fig. 4. Supercontinuum spectra generated in a a-Si:H core fiber pumped in the anomalous dispersion regime with $P_{0}=75 \mathrm{~W}$ and central wavelengths as labeled. The arrows indicate the positions of the 1st and 2nd order FWM sidebands (SB1 and SB2).

comparison to the predicted phase-matching curves, showing very good agreement. Furthermore, the results in Fig. 3 (b) indicate that with only $20 \mathrm{~W}$ peak power it is possible to tune the wavelength conversion over $600 \mathrm{~nm}$ ( $2 \mu \mathrm{m}$ to $2.6 \mu \mathrm{m}$ ), while only tuning the pump by $280 \mathrm{~nm}$. Further calculations have shown that by increasing the pump power to $\sim 60 \mathrm{~W}$ this could increase to $\sim 900 \mathrm{~nm}$.

By increasing the pump power even further, the FWM process can be used to seed supercontinuum generation. Fig. 4 shows the spectral broadening obtained for various pump wavelengths with a launch power of $75 \mathrm{~W}$. The top spectrum corresponds to the same pump wavelength as Fig. 3(a), however, in this case the generated spectral bandwidth is much broader (by $\sim 500 \mathrm{~nm}$ ). The shape of this spectrum suggests that the increased broadening is due to the appearance of a second set of sidebands positioned at $2 \Omega_{\max }$, associated with a higher order FWM process (see arrows). We attribute the ability to phase-match cascaded FWM processes to the high nonlinearity of the fiber that facilitates wavelength conversion over short propagation lengths, as is the case in this fiber. The remaining spectra in Fig. 4 have been obtained for increasingly longer pump wavelengths, where the linear and nonlinear losses are lower. In all cases these spectra exhibit more than an octave of continuum bandwidth at the $-30 \mathrm{~dB}$ level, with the total spectral coverage extending from $1.64 \mu \mathrm{m}$ to $3.37 \mu \mathrm{m}(\sim 1700 \mathrm{~nm})$. Interestingly, as well as an increase in the spectral bandwidth, the bottom two spectra exhibit an additional feature on the short wavelength edge characteristic of dispersive wave emission. Although dispersive waves are often associated with the onset of soliton fission, as the length of our fiber is shorter than the estimated fission length for the input pulses $\left(L_{\mathrm{fiss}} \simeq L_{\mathrm{D}} / N \sim 7 \mathrm{~mm}\right.$, where $L_{D}=T_{0}^{2} /\left|\beta_{2}\right| \sim 5 \mathrm{~cm}$ is the dispersion length and $N \sim 8$ is the soliton order), we attribute their appearance here to temporal break-up of the pulses caused by modulation induced via FWM [28], [29]. Further verification of this process is provided by the good agreement between the position of these short wavelength peaks centered at $\sim 1.8 \mu \mathrm{m}$ and the predicted wavelength of the dispersive wave emission $\lambda_{\mathrm{DW}} \sim 1.75 \mu \mathrm{m}$ as calculated via the phase-matching condition in Ref. [30].
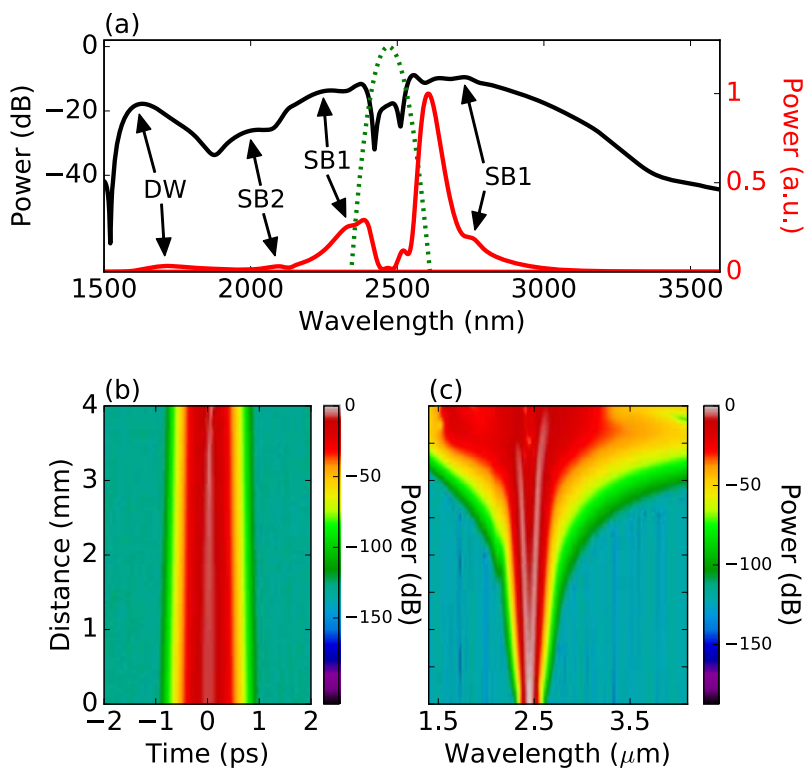

Fig. 5. (a) Simulated supercontinuum spectrum generated in a a-Si:H core fiber pumped at $2.47 \mu \mathrm{m}$ with $75 \mathrm{~W}$ input peak power (black) and input pulse spectrum (dashed green). The red spectrum is plotted on a linear scale (right-hand axis) to better resolve the position of the FWM side-bands. Temporal (b) and spectral (c) evolution of the optical pulses in the a-Si:H fiber.

To gain more insight into the underlying dynamics driving the supercontinuum generation in this material, numerical simulations based on a modified version of Eqs. (1) and (2) were performed, which also included Raman scattering and self steepening terms [28]. For a pump centered at $2.47 \mu \mathrm{m}$, the simulated waveguide parameters were chosen to match those experimentally measured in Ref. [3]. We set the linear loss to be $\alpha_{l}=0.8 \mathrm{~dB} / \mathrm{cm}$, nonlinear refractive index $n_{2}=1.04 \times 10^{-13} \mathrm{~cm}^{2} / \mathrm{W}$, carrier lifetime $\tau_{c}=87 \mathrm{~ns}$, $\beta_{\mathrm{TPA}}=0.02 \mathrm{~cm} / \mathrm{GW}$, and $A_{\mathrm{eff}}=1.23 \mu \mathrm{m}^{2}$. The dispersion parameters $\beta_{2}=-0.59 \mathrm{ps}^{2} / \mathrm{m}$ and $\beta_{3}=5.6 \times 10^{-3} \mathrm{ps}^{3} / \mathrm{m}$ were calculated from the Sellmeier equation for crystalline silicon raised by $\sim 4 \%$ to account for the higher refractive index of a-Si:H. We note that higher order dispersion terms were not included due to uncertainties in the material index and waveguide dimensions [31], and thus the simulated results at the spectral edges may only be qualitatively correct. Fig. 5(a) shows the simulated supercontinuum spectrum at the output of a $4 \mathrm{~mm}$ length of a-Si:H fiber for input pulses of $T_{\mathrm{FWHM}} \sim 300 \mathrm{fs}$ and $75 \mathrm{~W}$ input power. The corresponding temporal and spectral evolution of the pulse propagating in the a-Si:H fiber are shown in Fig. 5(b) and (c), respectively.

The numerical results of Fig. 5(a) are in reasonable agreement with the experimental spectrum in Fig. 4, in terms of the $-30 \mathrm{~dB}$ bandwidth $(1822 \mathrm{~nm}$ compared to $1700 \mathrm{~nm}$ in the experiment) and the appearance of several peaks around the pump wavelength. The first sidebands (SB1) appear at $2.32 \mu \mathrm{m}$ and $2.76 \mu \mathrm{m}$, close to both the theoretical predictions and experimental spectrum. In addition, a second sideband (SB2) is clearly visible at $2.09 \mu \mathrm{m}$ and a dispersive wave is generated around $1.71 \mu \mathrm{m}$, again matching the 

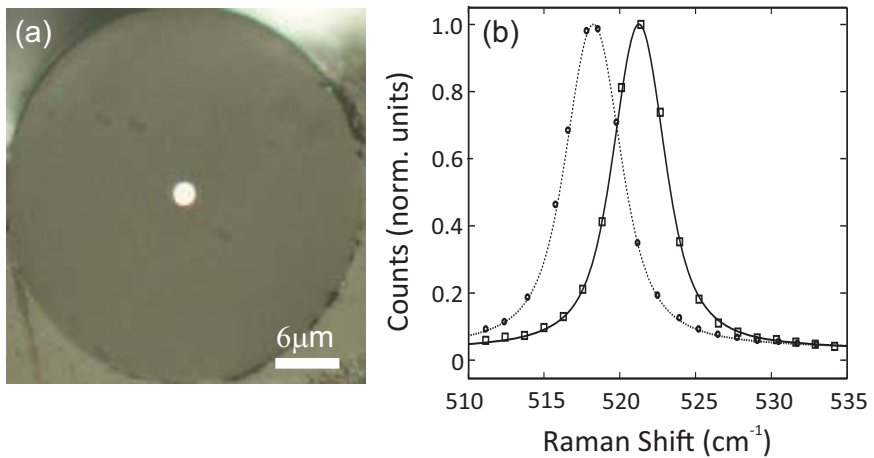

Fig. 6. (a) Microscope image of a tapered p-Si core fiber with a core diameter of $\sim 2 \mu \mathrm{m}$. (b) Raman spectrum of the $\mathrm{p}$-Si core (circles) compared to a single crystal wafer (squares). Fitted curves are used to estimate the line widths.

experimental observations and thus providing further confirmation of the dynamics described above. The temporal and spectral evolution of the pulse, shown in Fig. 5(b) and (c), indicate that most of the spectral broadening occurs after $3 \mathrm{~mm}$ of fiber. While the pulse shape in time remains fairly constant over the short fiber length considered here, the spectrum shows much richer dynamics. Most notably, the spectral peak at the pump laser wavelengths breaks into two peaks due to self-phase modulation (SPM). As the short-wavelength peak moves towards the zero-dispersion wavelength, and aided by the cascaded FWM sidebands, the dispersive wave is emitted. The dip in the spectrum at the original pump wavelength observed in the simulations, Fig. 5(a), is not found in the experiments (Fig. 4), which we attribute to light coupled into higher order modes: the fiber under consideration here supports $\sim 20$ modes but the simulations only consider the fundamental mode. We also note that the soliton fission lengths for the other pump wavelengths shown in Fig. 4 are significantly longer, which yields narrower broadening, also in agreement with the experiments.

\section{MCD Polycrystalline SiLicon Fibers}

Compared to the a-Si:H fibers, studies of nonlinear propagation in polycrystalline silicon ( $\mathrm{p}-\mathrm{Si}$ ) MCD fibers have been much more limited. This is due both to the larger core sizes of the as-drawn MCD fibers, where typical diameters are tens to hundreds of microns [7], [15], as well as the higher losses associated with scattering and absorption at the grain boundaries in the polycrystalline material [18]. To address these issues, several techniques have been developed to post-process the as-drawn fibers. Recently, the re-crystallisation of polycrystalline silicon fibers has been demonstrated using a continuous wave $\mathrm{CO}_{2}$ laser [32]. This technique allowed for the generation of centimeter long crystals, which reduced the linear optical losses from $15 \mathrm{~dB} / \mathrm{cm}$ to $2 \mathrm{~dB} / \mathrm{cm}$. However, the core diameters of these treated fibers remained too large for the observation of nonlinear propagation in this material. Thus, to overcome this limitation, a novel tapering procedure was developed [4], which had the benefit of both reducing the core size and improving the overall crystallinity by enhancing the grain growth and aligning the crystal orientation with respect to the fiber axis [33]. The values for the linear and nonlinear (a)
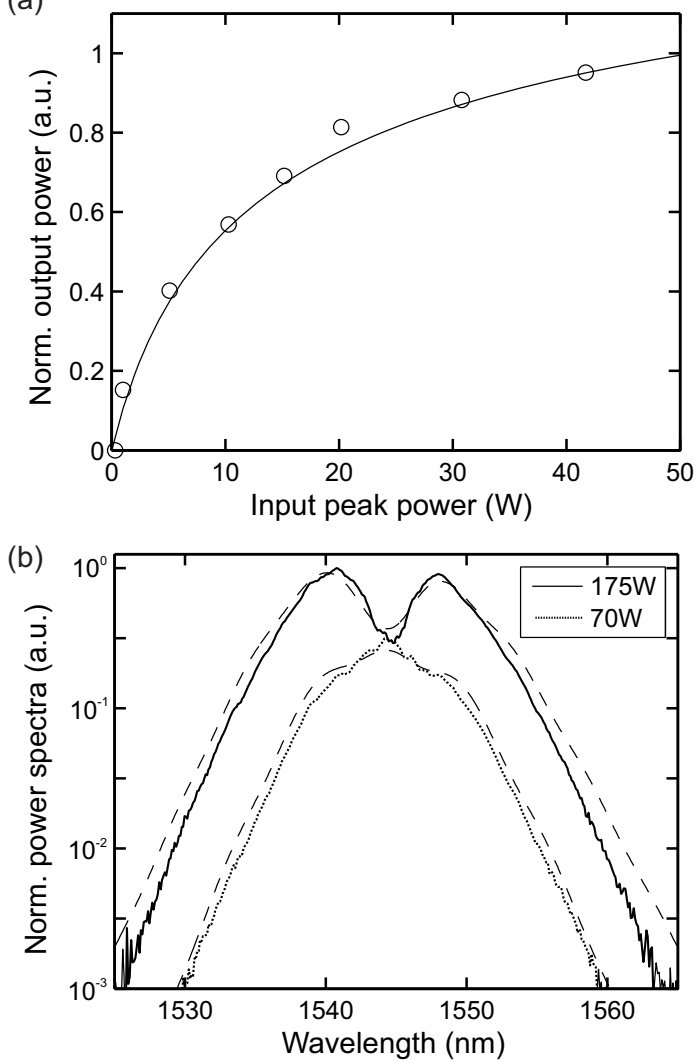

Fig. 7. (a) Nonlinear absorption as a function of coupled input peak power. The solid curve is the simulated fit to estimate $\beta_{\mathrm{TPA}}$. (b) Spectral broadening as a function of coupled peak power for two input powers (see legend). The dashed curves are simulated fits to estimate $n_{2}$.

properties of this material will thus depend on the averaged crystallinity, where we expect the key parameters to approach those of single crystal materials in the fibers with the largest grain sizes, i.e., when the volume of amorphous and defect material is at a minimum.

Using this tapering procedure, a number of low loss $(\sim 1-4 \mathrm{~dB} / \mathrm{cm})$ p-Si fibers have been demonstrated with core sizes in the range $900 \mathrm{~nm}-4 \mu \mathrm{m} \mathrm{[4],} \mathrm{[34].} \mathrm{Fig.} \mathrm{6(a)}$ shows a microscope image of a tapered $\mathrm{p}$-Si fiber with a core diameter of $\sim 2 \mu \mathrm{m}$ and Fig. 6(b) shows the corresponding Raman spectrum, as compared to a single crystal reference. The slight downshifting in the position of the fiber Raman peak is due to residual strain associated with differences in the thermal expansion of the core/cladding materials. Nevertheless, the excellent agreement in the Raman widths $\left(2.9 \mathrm{~cm}^{-1}\right.$ for the fiber compared to $2.7 \mathrm{~cm}^{-1}$ of the reference) indicates that the core is composed of high quality, large grain $\mathrm{p}-\mathrm{Si}$.

To demonstrate the suitability of these fibers for nonlinear propagation a set of high power transmission measurements were conducted, with the goal to benchmarking the values of the nonlinear refractive index and TPA parameter. As TPA manifests as an intensity dependent absorption, $\beta_{\text {TPA }}$ can be determined simply by monitoring the output power as a function of the coupled input power, as shown in Fig. 7(a). Subsequently, the size of $n_{2}$ can be estimated by mapping the spectral broadening due to selfphase modulation (SPM), shown in Fig. 7(b). In both cases the experimental data was obtained using a fiber with a core 
diameter of $D=2 \mu \mathrm{m}$, a length of $\sim 15 \mathrm{~mm}$, and a linear loss value of $\alpha_{l} \sim 2 \mathrm{~dB} / \mathrm{cm}$, which was pumped with a high power $1.54 \mu \mathrm{m}$ soliton source $\left(T_{\mathrm{FWHM}} \sim 700 \mathrm{fs}\right)$. The numerical fits were calculated using Eqs. (1) and (2), with the remaining material parameters such as the free carrier absorption and dispersion estimated from the single crystal values [19]. However, we note that the use of a femtosecond pump source ensures the role of the free carriers will be minimal, reducing their influence on our estimates. The results returned best fit values of $\beta_{\mathrm{TPA}} \sim 0.7 \mathrm{~cm} / \mathrm{GW}$ and $n_{2} \sim 5 \times 10^{-14} \mathrm{~cm}^{2} / \mathrm{W}$, which are both within the range previously reported for single crystal silicon [35]. Table 2 summarizes the key linear and nonlinear parameters measured in a selection of tapered MCD p-Si fibers with the lowest losses, which we expect to consist of the largest crystal grains. Significantly, these measurements represent the first reports of nonlinear propagation in a p-Si waveguide, either fiber or planar-based, which provides further evidence of the high material quality of the post-processed fibers.

TABLE 2

Material properties for tapered p-Si core fibers.

\begin{tabular}{|c|c|}
\hline Pump $\lambda$ & $1.55 \mu \mathrm{m}$ \\
\hline \hline$\alpha_{l}(\mathrm{~dB} / \mathrm{cm})$ & $1.6-3$ \\
\hline$n_{2}\left(\times 10^{-14} \mathrm{~cm}^{2} / \mathrm{W}\right)$ & $4-6$ \\
\hline$\beta_{\mathrm{TPA}}(\mathrm{cm} / \mathrm{GW})$ & $0.7-1.0$ \\
\hline
\end{tabular}

As these first characterization measurements were performed in the telecoms band where the material dispersion is normal, observations of FWM and/or broadband continuum generation have yet to be made in these fibers. However, as the key benefit of the MCD fibers is their long lengths, we have been able to fabricate p-Si tapers with micrometer sized cores over lengths of $\sim 2-3 \mathrm{~cm}$, which is considerably longer than the few millimeter lengths more typical of HPCVD fibers used in optical experiments [3]. Thus, in order to compare the suitability of these fibers for supercontinuum generation against the HPCVD fibers, numerical investigations similar to those of Fig. 5 have been performed. The fiber parameters were based on a tapered p-Si structure with a $2 \mu \mathrm{m}$ core diameter and a length of $13 \mathrm{~mm}$. With the pump pulses positioned at $2.42 \mu \mathrm{m}$, we can assume: $\alpha \sim 1 \mathrm{~dB} / \mathrm{cm}, n_{2}=5.15 \times 10^{-14} \mathrm{~cm}^{2} / \mathrm{W}$, $\tau_{c}=87 \mathrm{~ns}, \beta_{\mathrm{TPA}}=0.02 \mathrm{~cm}^{2} / \mathrm{GW}, A_{\text {eff }}=1.68 \mu \mathrm{m}^{2}$ and the calculated dispersion parameters of $\beta_{2}=-0.23 \mathrm{ps}^{2} / \mathrm{m}$ and $\beta_{3}=4 \times 10^{-3} \mathrm{ps}^{3} / \mathrm{m}$.

The simulation results are shown in Fig 8. Similar to the a-Si:H fiber, the spectral broadening is dominated by SPM, cascaded FWM, and the emission of a dispersive wave. The first sidebands (SB1) are calculated to occur at $2.2 \mu \mathrm{m}$ and $2.66 \mu \mathrm{m}$, and appear in the simulated data at $2.26 \mu \mathrm{m}$ and $2.69 \mu \mathrm{m}$, respectively. The second sidebands (SB2) are predicted at $2.04 \mu \mathrm{m}$ and $2.96 \mu \mathrm{m}$. As in the previous case, only the short wavelength SB2 is observable at $2.1 \mu \mathrm{m}$. As expected, a first dispersive wave is emitted at $1.95 \mu \mathrm{m}$, but not until $11 \mathrm{~mm}$ of propagation owing to the lower nonlinear index of this material. The dispersive wave does, however, shift out further to $1.8 \mu \mathrm{m}$ at the end of the fiber, resulting in a $-30 \mathrm{~dB}$ spectral bandwidth of $1460 \mathrm{~nm}$.
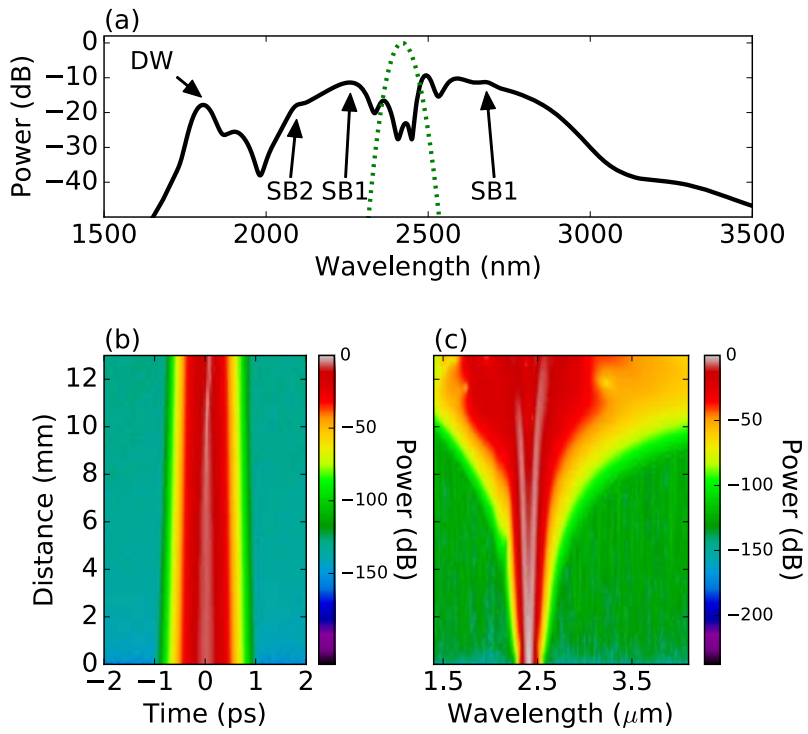

Fig. 8. (a) Simulated supercontinuum spectrum generated in a p-Si core fiber pumped at $2.42 \mu \mathrm{m}$ with $75 \mathrm{~W}$ input power (black) and input pulse spectrum (dashed green). Temporal (b) and spectral (c) evolution of the optical pulses in the p-Si fiber.

Although this broadening is below what was obtained in the a-Si:H fiber, and less than an octave, it could be increased quite significantly by reducing the losses and increasing the taper lengths as this would allow for propagation beyond the soliton fission length. Experimental efforts in this area are currently ongoing.

\section{Future Challenges}

The nonlinear measurements of the silicon core fibers described above represent the first steps towards the development of new wavelength sources using this technology. However, there are still a number of challenges that must be overcome to improve the efficiency of these systems, as outlined in this section.

\subsection{Dispersion control}

The nonlinear performance of the semiconductor core fiber devices ultimately depends on the fiber geometry and material properties; specifically the Kerr nonlinearity (nonlinear index and loss) and dispersion. It has already been shown that the optical propagation of silicon core fibers can be modified by external treatments such as the inscription of Bragg gratings [36], and such structures could also be used for cavity enhancement of the nonlinear processes. Alternatively, more complex material systems could enable new possibilities. For example, the addition of germanium into silicon can extend the transmission further into the midinfrared, increase the nonlinear coefficients [37], and permit spatial variations in the alloy concentration that can tailor dispersion. In recent work to model SiGe planar waveguides [38], it has been demonstrated that spatial control of the germanium content can lead to flat dispersion characteristics. Furthermore, higher optical intensities are predicted in the regions of the waveguide with higher germanium 

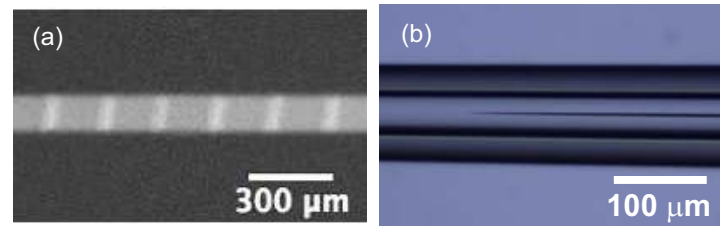

(c)

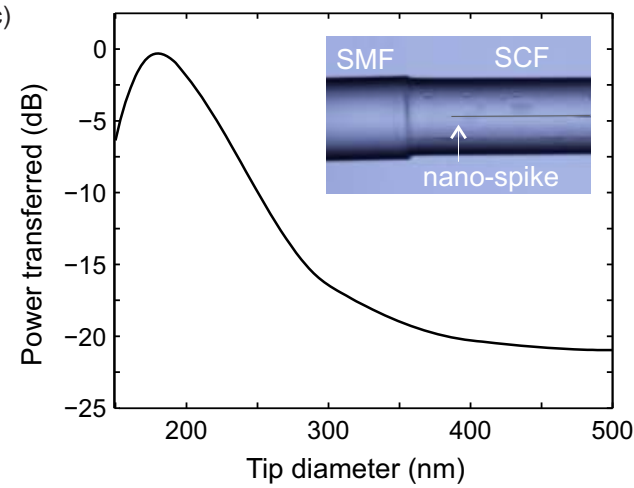

Fig. 9. (a) XCT image of a Ge-rich grating formed in a SiGe fiber core by laser processing. (b) Microscope image of a silicon nano-spike for efficient input coupling. (c) Mode overlap between the silicon nanospike and a standard SMF as a function of tip diameter. Inset shows the spliced SMF-silicon nano-spike device.

concentrations (due to the larger index of refraction), and the larger $n_{2}$ of germanium, relative to silicon, then leads to larger nonlinear effects. Although SiGe-core fibers have only recently been realized, control over the spatial concentration of the germanium has already been demonstrated via a $\mathrm{CO}_{2}$ laser treatment (see Fig. 9(a) [39]), which opens up additional process variables for nonlinear device design. The use of III-V core materials would further extend the range of nonlinear devices possible.

\subsection{Interconnection}

Despite their physical similarity to single mode fibers (SMFs), coupling light efficiently into and out of the silicon core fibers remains a difficult task due to silicon's high refractive index, which results in significant reflection losses and a large mode mismatch. A few solutions have been proposed to overcome this integration challenge, including the use of microstructured fiber designs to better match the mode area [40] and chemical etchants to reduce the reflection at the interface [16], though so far no method has addressed both issues simultaneously. Thus a promising alternative solution would be to adopt the well-established inverse taper approach widely employed by the planar community [41]. This method has also gained some attention within the novel material fiber community where a tapered nano-spike was used to improve coupling into a chalcogenide core fiber [42]. Here, the key idea is that by decreasing the high index core to nanoscale dimensions at the facet, the guided mode spreads out to better match both the area and the effective index of the SMF mode.

To demonstrate this, the tapering procedure described in Section 5 has been adapted to fabricate nano-spikes on the end of high quality p-Si fibers as shown in Fig. 9(b) [43]. These spikes can be cleaved and spliced to standard SMF using a modified fusion splicing technique to obtain a robust joint (see inset in Fig. 9(c)). Optical transmission measurements of the integrated silicon fiber-SMF device have revealed insertion losses of $\sim 7 \mathrm{~dB}$. Although a promising preliminary result, the relatively high losses of this system can be attributed to non-optimized dimensions of the nanospike and a slight misalignment of the spliced fiber cores. Indeed, numerical simulations of the mode overlap at the splice joint calculated as a function of the tip diameter, as shown in Fig. 9(c), indicate that the minimum transmission loss between the two fibers could be as low as $\sim 0.5 \mathrm{~dB}$ for an optimized tip diameter of $190 \mathrm{~nm}$. Clearly, such low integration losses would greatly improve the efficiency and practicality of the nonlinear systems.

\section{CONCLUSION}

This paper has reported the recent advances in wavelength conversion and supercontinuum generation in silicon core fibers. Results have been presented for both HPCVD deposited hydrogenated amorphous silicon fibers and MCD polycrystalline silicon fibers. Cascaded four-wave mixing and octave spanning supercontinum generation have been demonstrated in the infrared region in the HPCVD fibers. To date, the demonstration of nonlinear effects in the MCD fibers lags behind those of the HPCVD fibers, primarily because of their lower nonlinearity, larger core size, and higher losses. However, numerical simulations suggest that similar spectral broadening is achievable in longer length tapered MCD fibers. Although, as yet, the spectral broadening observed in the silicon fibers is narrower than what has been reported in alternative materials such as ZBLAN fibers [44], the fiber lengths and input powers required for supercontinuum generation in the silicon cores are several orders of magnitude smaller. Continued advancements to reduce the material losses and improve the device efficiency will open the door to many new and exciting possibilities in nonlinear device development.

\section{ACKNOWLEDGMENT}

The authors would like to thank their colleagues and collaborators who contributed to the cited work. This work was financially supported by EPSRC [EP/J004863/1, EP/P000940/1]; RCN [NorFab 197411/V30]; NTNU and the Norwegian Research Council [grant 262232]; Northrop Grumman Corporation. The data for this paper are accessible through the University of Southampton Institutional Research Repository (DOI:10.5258/SOTON/D0265).

\section{REFERENCES}

[1] A. C. Peacock, U. J. Gibson, and J. Ballato, "Silicon optical fibres - past, present, and future," Advances in Physics: X, vol. 1, p. 114, 2016.

[2] N. Vukovic, N. Healy, F. H. Suhailin, P. Mehta, T. D. Day, J. V. Badding, and A. C. Peacock, "Ultrafast optical control using the Kerr nonlinearity in hydrogenated amorphous silicon microcylindrical resonators," Sci. Rep., vol. 3, p. 2885, 2013.

[3] L. Shen, N. Healy, L. Xu, H. Y. Cheng, T. D. Day, J. H. V. Price, J. V. Badding, and A. C. Peacock, "Four-wave mixing and octave-spanning supercontinuum generation in a small core hydrogenated amorphous silicon fiber pumped in the mid-infrared," Opt. Lett., vol. 39, p. 5721, 2014.

[4] F. H. Suhailin, L. Shen, N. Healy, L. Xiao, M. Jones, T. Hawkins, J. Ballato, U. J. Gibson, and A. C. Peacock, "Tapered polysilicon core fibers for nonlinear photonics," Opt. Lett., vol. 41, p. 1360, 2016. 
[5] S. Morris, T. Hawkins, P. Foy, J. Ballato, S. W. Martin, and R. Rice, "Cladding glass development for semiconductor core optical fibres," Int. J. Appl. Glass. Sci., vol. 3, p. 144, 2012.

[6] L. Lagonigro, N. Healy, J. R. Sparks, N. F. Baril, P. J. A. Sazio, J. V. Badding, and A. C. Peacock, "Low loss silicon fibers for photonic applications," Appl. Phys. Lett., vol. 96, p. 041105, 2010.

[7] J. Ballato, T. Hawkins, P. Foy, R. Stolen, B. Kokuoz, M. Ellison, C. McMillen, J. Reppert, A. M. Rao, M. Daw, S. Sharma, R. Shori, O. Stafsudd, R. R. Rice, and D. R. Powers, "Silicon optical fiber," Opt. Express, vol. 16, p. 18675, 2008.

[8] P. J. A. Sazio, A. Amezcua-Correa, C. E. Finlayson, J. R. Hayes, T. J Scheidemantel, N. F. Baril, B. R. Jackson, D.-J. Won, F. Zhang, E. R. Margine, V. Gopalan, V. H. Crespi, and J. V. Badding, "Microstructured optical fibers as high-pressure microfluidic reactors," Science, vol. 311, p. 1583, 2006.

[9] P. J. Roberts, F. Couny, H. Sabert, B. J. Mangan, D. P. Williams, L. Farr, M. W. Mason, A. Tomlinson, T. A. Birks, J. C. Knight, and P. S. J. Russell, "Ultimate low loss of hollow-core photonic crystal fibres," Opt. Express, vol. 13, p. 236, 2005.

[10] N. Healy, L. Lagonigro, J. R. Sparks, S. Boden, P. J. A. Sazio, J. V. Badding, and A. C. Peacock, "Polycrystalline silicon optical fibers with atomically smooth surfaces," Opt. Lett., vol. 36, p. 2480, 2011.

[11] N. F. Baril, R. He, T. D. Day, J. R. Sparks, B. Keshavarzi, M. Krishnamurthi, A. Borhan, V. Gopalan, A. C. Peacock, N. Healy, P. J. A. Sazio, and J. V. Badding, "Low loss silicon fibers for photonic applications," J. Am. Chem. Soc., vol. 134, p. 19, 2011.

[12] S. Chaudhuri, J. R. Sparks, X. Ji, M. Krishnamurthi, L. Shen, N. Healy, A. C. Peacock, V. Gopalan, and J. V. Badding, "Crystalline silicon optical fibers with low optical loss," ACS Photonics, vol. 3, p. 378384, 2016.

[13] J. Ballato and E. Snitzer, "Fabrication of fibers with high rareearth concentrations for faraday isolator applications," Appl. Opt., vol. 34, p. 6848, 1995.

[14] B. Scott, W. Ke, and G. Pickrell, "Fabrication of n-type silicon optical fibers," IEEE Photon. Technol. Lett., vol. 21, p. 1798, 2009

[15] E. Nordstrand, A. Dibbs, A. Eraker, and U. J. Gibson, "Alkaline oxide interface modifiers for silicon fiber production," Opt. Mater. Express, vol. 3, p. 651, 2013.

[16] J. Chen, Y. Sun, and L. A. Wang, "Reducing splicing loss between a silicon-cored optical fiber and a silica optical fiber," IEEE Photon. Technol. Lett., vol. 28, p. 1774, 2016.

[17] J. Ballato, T. Hawkins, P. Foy, S. Morris, N. K. Hon, B. Jalali, and R. Rice, "Silica-clad crystalline germanium core optical fibers," Opt. Lett., vol. 36, p. 687, 2011.

[18] B. L. Scott and G. R. Pickrell, "Silicon optical fiber diameter dependent grain size," J. Cryst. Growth, vol. 371, p. 134, 2013.

[19] L. Yin and G. Agrawal, "Impact of two-photon absorption on selfphase modulation in silicon waveguides," Opt. Lett., vol. 32, p. 2031, 2007.

[20] J. Leuthold, C. Koos, and W. Freude, "Nonlinear silicon photonics," Nat. Photonics, vol. 4, p. 535, 2010.

[21] P. Mehta, N. Healy, N. F. Baril, P. J. A. Sazio, J. V. Badding, and A. C. Peacock, "Nonlinear transmission properties of hydrogenated amorphous silicon core optical fibers," Opt. Express, vol. 18, p. 16826, 2010.

[22] K. Narayanan and S. F. Preble, "Broadband all-optical modulation in hydrogenated-amorphous silicon waveguides," Opt. Express, vol. 18, p. 8998, 2010.

[23] L. Shen, N. Healy, P. Mehta, T. D. Day, J. R. Sparks, J. V. Badding, and A. C. Peacock, "Nonlinear transmission properties of hydrogenated amorphous silicon core fibers towards the mid-infrared regime," Opt. Express, vol. 21, p. 13075, 2013.

[24] C. Grillet, L. Carletti, C. Monat, P. Grosse, B. B. Bakir, S. Menezo, J. M. Fedeli, and D. J. Moss, "Amorphous silicon nanowires combining high nonlinearity, FOM and optical stability," Opt. Express, vol. 20, p. 22609, 2012

[25] P. Mehta, N. Healy, J. R. Sparks, T. D. Day, P. J. A. Sazio, J. V. Badding, and A. C. Peacock, "All-optical modulation using twophoton absorption in silicon core optical fibers," Opt. Express, vol. 19, p. 19078, 2011.

[26] P. Mehta, N. Healy, T. D. Day, J. V. Badding, and A. C. Peacock, "Ultrafast wavelength conversion via cross-phase modulation in hydrogenated amorphous silicon optical fibers," Opt. Express, vol. 20, p. 26110, 2012.

[27] G. P. Agrawal, Fiber-Optic Communication Systems, 4th Edition. Wiley, 2010.
[28] G. Genty, S. Coen, and J. M. Dudley, "Fiber supercontinuum sources," J. Opt. Soc. Am. B, vol. 24, p. 1771, 2007.

[29] D. Castelló-Lurbe, N. Vermeulen, and E. Silvestre, "Towards an analytical framework for tailoring supercontinuum generation," Opt. Express, vol. 24, p. 26629, 2016.

[30] L. Yin, Q. Lin, and G. P. Agrawal, "Soliton fission and supercontinuum generation in silicon waveguides," Opt. Lett., vol. 32, p. 391, 2007.

[31] U. D. Dave, S. Uvin, B. Kuyken, S. S. F. Leo, and G. Roelkens, "Telecom to mid-infrared spanning supercontinuum generation in hydrogenated amorphous silicon waveguides using a thulium doped fiber laser pump source," Opt. Express, vol. 21, p. 32032, 2013.

[32] N. Healy, M. Fokine, Y. Franz, T. Hawkins, M. Jones, J. Ballato, A. C. Peacock, and U. J. Gibson, " $\mathrm{CO}_{2}$ laser-induced directional recrystallization to produce single crystal silicon-core optical fibers with low loss," Adv. Optical Mater., vol. DOI: 10.1002/adom.201500784, 2016.

[33] C. McMillen, G. Brambilla, S. Morris, T. Hawkins, P. Foy, N. Broderick, E. Koukharenko, R. Rice, and J. Ballato, "On crystallographic orientation in crystal core optical fibers ii: Effects of tapering," Opt. Mat., vol. 35, p. 93, 2012.

[34] Y. Franz, A. F. J. Runge, N. H. H. Ren, K. Ignatyev, M. Jones, T. Hawkins, J. Ballato, U. J. Gibson, and A. C. Peacock, "Material properties of tapered crystalline silicon core fibers," Opt. Mat Express, vol. 7, p. 2055, 2017.

[35] A. D. Bristow, N. Rotenberg, and H. M. van Driel, "Two-photon absorption and kerr coefficients of silicon for 850-2200 nm," Appl. Phys. Lett., vol. 90, p. 191104, 2007.

[36] M. Fokine, A. Theodosiou, S. Song, T. Hawkins, J. Ballato, K. Kalli, and U. J. Gibson, "Laser structuring, stress modification and bragg grating inscription in silicon-core glass fibers," Opt. Mater. Express, vol. 7, p. 1589, 2017.

[37] N. K. Hon, R. Soref, and B. Jalali, "The third-order nonlinea optical coefficients of $\mathrm{Si}, \mathrm{Ge}$, and $\mathrm{Si}_{(1-x)} \mathrm{Ge}_{x}$ in the midwave and longwave infrared," J. Appl. Phys., vol. 110, p. 011301, 2011.

[38] J. M. Ramirez, V. Vakarin, J. Frigerio, P. Chaisakul, D. Chrastina, X. L. Roux, A. Ballabio, L. Vivien, G. Isella, and D. Marris-Morini, "Ge-rich graded-index $\mathrm{Si}_{(1-x)} \mathrm{Ge}_{x}$ waveguides with broadband tight mode confinement and flat anomalous dispersion for nonlinear mid-infrared photonics," Opt. Express, vol. 25, p. 6561, 2017.

[39] D. A. Coucheron, M. Fokine, N. Patil, D. W. Breiby, O. T. Buset, N. Healy, A. C. Peacock, T. Hawkins, M. Jones, J. Ballato, and U. J. Gibson, "Laser recrystallization and inscription of compositional microstructures in crystalline SiGe-core fibres," Nat. Commun., vol. 7, p. 13265, 2016

[40] N. Healy, J. R. Sparks, M. N. Petrovich, P. J. A. Sazio, J. V. Badding, and A. C. Peacock, "Large mode area silicon microstructured fiber with robust dual mode guidance," Opt. Express, vol. 17, p. 18076, 2009.

[41] V. R. Almeida, R. R. Panepucci, and M. Lipson, "Nanotaper for compact mode conversion," Opt. Lett., vol. 28, p. 1302, 2003.

[42] N. Granzow, M. A. Schmidt, W. Chang, L. Wang, Q. Coulombier, J. Troles, P. Toupin, I. Hartl, K. F. Lee, M. E. Fermann, L. Wondraczek, and P. S. J. Russell, "Mid-infrared supercontinuum generation in $\mathrm{As}_{2} \mathrm{~S}_{3}$-silica "nanospike" step-index waveguide," Opt. Express, vol. 21, p. 10969, 2013.

[43] H. Ren, A. F. J. Runge, J. Campling, M. Jones, T. Hawkins, J. Ballato, P. Horak, U. Gibson, and A. C. Peacock, "Silicon fibre nano-spike for robust coupling to silica fibres," CLEO-Europe, vol. CK-9.2, 2017.

[44] A. M. Heidt, J. H. V. Price, C. Baskiotis, J. S. Feehan, Z. Li, S. U. Alam, and D. J. Richardson, "Mid-infrared zblan fiber supercontinuum source using picosecond diode-pumping at $2 \mu \mathrm{m}$," Opt. Express, vol. 21, p. 24281, 2013.

A. C. Peacock (M'14-SM'15) is a Professor of Photonics within the Optoelectronics Research Centre (ORC) at the University of Southampton. She obtained her BSc and MSc in Physics from The University of Auckland (New Zealand) in 1999 and 2001, respectively, and her PhD from the ORC in 2004. In 2007 she was awarded a five-year Royal Academy of Engineering Research Fellowship, following which she established the Nonlinear Semiconductor Photonics group, where the focus of the research is on the design and development of novel semiconductor 
waveguides. She is a fellow of The Optical Society (FOSA) and the Institute of Physics (FInstP) and currently holds a five-year EPSRC research fellowship.

J. Campling is a PhD student at the Optoelectronics Research Centre (ORC) working on numerical modelling of nonlinear pulse propagation in semiconductor fibers. He gained an MSc in Frontiers of Quantum Technology at the University of Sussex in 2016 and a BSc in Physical Sciences from the Open University in 2014.

A. F. J. Runge is a research fellow at the Optoelectronics Research Centre (ORC) at the University of Southampton. He received his BSc and MSc in Physics from the Université de Franche-Comté in 2009 and 2011, respectively. He then obtained his PhD from the University of Auckland in 2015. His research focuses on nonlinear semiconductor photonics and laser processing of semiconductor materials.

H. Ren joined the Optoelectronics Research Centre (ORC) at University of Southampton as a PhD student in 2015. He completed his BEng Electrical Engineering \& Electronics from University of Edinburgh and Xiamen University in 2015. His current research interests include nonlinear semiconductor photonics and tapered silicon core fibers.

L. Shen received the BSc. and M.Phil. degrees from Huazhong University of Science and Technology (HUST), Wuhan, China, in 2009 and 2012, respectively. Then obtained his PhD degree from the Optoelectronics Research Centre (ORC), University of Southampton, in 2015. He is currently a lecturer at HUST and a visiting scholar at the ORC. His research interests include novel semiconductor photonic devices.

O. Aktas is a postdoctoral research fellow within the Optoelectronics Research Centre (ORC) at the University of Southampton. He obtained his BSc in Electrical and Electronics Engineering in 2005 from the Ankara University (Turkey) and then worked as a military engineer in the Turkish Aviation Industry for 10 years. During this period, he earned his MSc and PhD in Physics from the Bilkent University (Turkey) in 2008 and 2014, respectively. In his PhD thesis, he developed a new top-tobottom nanotechnology fabrication method based on fiber drawing of chalcogenides, and demonstrated photonic applications for photoconductive nanowires, optical microcavity WGM resonators and tapered fibers. He published several articles in the top ranked journals in the fields of Material Science, Nanotechnology, Physics and Photonics.

P. Horak received his MSc in theoretical physics and $\mathrm{PhD}$ in theoretical quantum optics from the University of Innsbruck, Austria, in 1993 and 1997, respectively. Since 2001, he has been with the Optoelectronics Research Centre, University of Southampton, where he now holds the position of Associate Professor and heads the Computational Nonlinear Optics group. $\mathrm{He}$ is interested in the theoretical and numerical investigation of photonic devices, including linear, nonlinear, and quantum optical effects.
N. Healy is a Senior Lecturer of Physics at Newcastle University. He is the Head of the Emerging Technologies and Materials Group and Director of the Photon Science Laboratory. He obtained his PhD from The University of Limerick (Ireland) 2008. His research interests lie in the areas of semiconductor photonics, high intensity light matter interactions, and optical fiber processing for devices.

U. J. Gibson is a professor of physics at the Norwegian University of Science and Technology (NTNU), Norway and holds adjunct positions in Applied Physics at the KTH Royal Institute of Technology in Stockholm and the Chemistry Department of Dartmouth College in New Hampshire. Gibson received her A.B. from Dartmouth and the Ph.D. in physics from Cornell University in 1982. She then held assistant and associate professorships at the University of Arizona's Optical Sciences Center in Tucson, Arizona. In 1990, she moved to Dartmouth's Thayer School of Engineering, Hanover, New Hampshire. She has more than 100 refereed journal articles, 7 book chapters, and three US Patents. In 2008 Gibson held a Fulbright Scholarship, working at VTT research labs in Espoo, FInland. In 2016, she was elected to the leadership of OSA, where she currently holds the Vice-presidential position.

J. Ballato (M'97-SM'12-F'17) is a professor of materials science and engineering at Clemson University where he is the inaugural holder of the Sirrine Endowed Chair in Optical Fiber. He earned a B.S. in Ceramic Science and Engineering (1993) and the Ph.D. in Ceramic and Materials Engineering (1997) from Rutgers, The State University of New Jersey. He has published more than 350 technical papers and holds 34 U.S. and foreign patents. Among numerous other honors, his collaborative work on Anderson-localizing optical fiber was chosen as one of Physics World's Top Ten Breakthroughs for 2014. He is a Fellow of the Institute of Electrical and Electronics Engineers (IEEE), the Optical Society of America (OSA), the International Society of Optical Engineering (SPIE), and the American Ceramic Society (ACerS) as well as being an elected member of the World Academy of Ceramics and the US National Academy of Inventors. 